\title{
Profundización teórica de modelos de volatilidad ARCH - GARCH y una aplicación al caso colombiano
}

\author{
Deepening the theorical volatility models ARCH - GARCH and an \\ application to the Colombian case
}

Heivar Yesid Rodríguez Pinzóna

heivarrodriguez@usantotomas.edu.co

\section{Resumen}

Con este trabajo se quiere presentar el soporte teórico de los modelos ARCH y GARCH propuestos por Engle (1982) y Bollerslev (1986), desarrollando las demostraciones de media y varianza, condicional y no-condicional a partir de los supuestos hechos por Engle (1982) y finalmente se presenta el ajuste de un proceso, que presenta dinámicas ARCH y GARCH.

Palabras clave: modelos ARCH, modelos GARCH, volatilidad, inflación.

\begin{abstract}
This work presents the theoretical support of ARCH and GARCH models proposed by Engle (1982) and Bollerslev (1986), developing the demonstrations of mean and variance, conditional and non-conditional based in the assumptions made by Engle (1982) and finally it presents an adjustment process, which presents the dynamic proper of ARCH and GARCH models.
\end{abstract}

Key words: ARCH models, GARCH models, volatility, inflation.

\section{Introducción}

Los desarrollos de series de tiempo, propuestos por Box \& Jenkins (1976), plantean el modelamiento la serie en nivel, olvidando en parte la volatililidad del proceso del cual proviene la misma, característica que en la actualidad, se ha convertido en un elemento clave en la toma de decisiones, especialmente en mercados financieros.

${ }^{a}$ Docente. Universidad Santo Tomás 
En estos mercados la volatilidad es una característica fundamental, su medición y pronóstico es de vital importancia para los operadores para asegurarse o apalancarse frente a eventos que conlleven aperdidas que no se puedan cubrir. De manera que la ausencia de un análisis de la volatilidad debilita todo proceso de toma de decisiones.

Al considerar la volatilidad como un proceso estocástico, se buscar ajustar un modelo que permita describir y analizar su comportamiento histórico y a partir de este su comportamiento futuro. Para el caso de procesos de varianza constante la metodología de Box-Jenkins ha sido ampliamente utilizada, sin embargo, este supuesto no es sostenible (plausible) en varias áreas de investigación, por lo que se deben consideran otras alternativas.

Dentro de estas alternativas aparecen los modelos ARCH (Autorregresive Condicional Heterocedastic) y GARCH (Generalized Autorregresive Condicional Heterocedastic) propuestos por Engle (1982) y Bollerslev (1986) respectivamente, modelos que permiten especificar el comportamiento de la varianza. Estos modelos se plantean pensando que se modela en la media condicional y la varianza condicional simultáneamente. La diferencia entre lo condicional y no condicional es que la expectativa condicional se refiere a una expectativa hacia el futuro pero sujeta a la información observada hasta el momento $t$ - corto plazo -.

Este trabajo presenta el soporte teórico de los modelos ARCH y GARCH propuestos por Engle (1982) y Bollerslev (1986). Se desarrollan las demostraciones de varianza condicional y no-condicional a partir de los supuestos hechos por Engle (1982) y finalmente se presenta una apliación la serie de la Inflación Colombiana.

\section{Modelos}

Los modelos presentados plantean la varianza $h_{t}$ - volatilidad-, como proceso estocástico que obedece un modelo a estimar, los principales resultados de los artículos de Engle (1982) y Bollerslev (1986) son mostrados en las secciones 2.1 y 2.2 y posteriormente en la sección 3 , se presentan los resultados de este trabajo, y son las demostraciones no desarrolladas en los artículos antes mencionados.

\subsection{Modelo Autorregresivo Condicional Heterocedástico (ARCH)}

El modelo ARCH fue presentado por Engle (1982), como alternativa para modelar procesos con periodos de turbulencia y periodos de calma, y a largo plazo sigan siendo estacionarios. La familia de procesos $\mathrm{ARCH}$ esta definida como se muestra a continuación.

Un proceso $\left\{y_{t}\right\}$ es un proceso $\mathrm{ARCH}(\mathrm{p})$ si obedece las ecuaciones:

$$
y_{t}=z_{t} h_{t}^{1 / 2} \quad \text { con } z_{t} \sim N I D(0,1)
$$

Comunicaciones en Estadística, junio 2009, Vol. 2, No. 1 


$$
h_{t}=\alpha_{0}+\sum_{i=1}^{p} \alpha_{i} y_{t-i}^{2}, \quad \text { con } \alpha_{0}>0 \quad \text { y } \alpha_{i} \geq 0 \quad \text { para } i=1, \ldots, p
$$

A partir de estos supuestos se puede mostrar:

1. $E\left(y_{t}\right)=0$

2. $V\left(y_{t}\right)=\frac{\alpha_{0}}{1-\sum_{i=1}^{p} \alpha_{i}}$

Nota 1: De 2., se tiene que $\sum_{i=1}^{p} \alpha_{i}<1$.

\subsection{Modelo Autorregresivo Condicional Heterocedástico Ge- neralizado (GARCH)}

Este proceso fue presentado por Bollerslev (1986); es una generalización de los procesos ARCH. El modelo GARCH adiciona una parte autoregresiva al comportamiento de la varianza, planteado en el modelo ARCH.

Un proceso $\left\{y_{t}\right\}$ obedece una dinámica $\operatorname{GARCH}(\mathrm{p}, \mathrm{q})$, si satisface las ecuaciones:

$$
\begin{gathered}
y_{t}=z_{t} h_{t}^{1 / 2}, z_{t} \sim \operatorname{NID}(0,1), h_{t}>0 \quad \mathrm{y} z_{t} \perp y_{s}(s<t) \\
h_{t}=\alpha_{0}+\sum_{j=1}^{q} \alpha_{j} y_{t-j}^{2}+\sum_{i=1}^{p} \beta_{i} h_{t-i}, \quad \operatorname{con} p \geq 0, q>0, \alpha_{0}>0, \\
\alpha_{j} \geq 0, i=1, \ldots, q, \quad \mathrm{y} \beta_{i} \geq 0, i=1, \ldots, p
\end{gathered}
$$

Notese que $z_{t} \perp y_{s}$ equivale a $\operatorname{Cov}\left(z_{t}, y_{s}\right)=0$ para todo $(s<t)$.

Dados los supuestos anteriores se tiene:

1. $E\left(y_{t}\right)=0$

2. $V\left(y_{t}\right)=\frac{\alpha_{0}}{1-\sum_{j=1}^{q} \alpha_{j}-\sum_{i=1}^{p} \beta_{i}}$

Nota 2: Analogo a lo mostrado para un porceso $\mathrm{ARCH}(\mathrm{p})$ se tiene que,

$$
\sum_{j=1}^{q} \alpha_{j}+\sum_{i=1}^{p} \beta_{i}<1
$$




\section{Resultados}

Los procesos ARCH y GARCH planteados en el capitulo anterior presentan características importantes para el modelado de series de tiempo y para un mejor entendimiento del comportamiento asociado a este tipo de procesos. A continuación se presentan las demostraciones para llegar a sus momentos de orden 1 y 2 , a partir de los supuestos dados en las ecuaciones (1) al (4). Inicialmente los resultados se presentan para un proceso $\mathrm{ARCH}(1)$, en seguida para un $\mathrm{ARCH}(\mathrm{p})$, y finalmente para un proceso $\operatorname{GARCH}(1,1)$.

\subsection{Proceso $\mathrm{ARCH}(1)$}

\subsubsection{Esperanza y varianza no-condicionales}

De las ecuaciones (1) y (2) tenemos $y_{t}=z_{t} h_{t}^{1 / 2}$ con $z_{t} \sim N I D(0,1)$ y $h_{t}=$ $\alpha_{0}+\alpha_{1} y_{t-1}^{2}$.

El primer y segundo Momento No-condicionales, del proceso $y_{t}$, están dados por:

1. Media

$$
\begin{aligned}
E\left(y_{t}\right) & =E\left(z_{t} h_{t}^{1 / 2}\right) \\
& =E\left(z_{t}\right) E\left[\left(\alpha_{0}+\alpha_{1} y_{t-1}^{2}\right)^{1 / 2}\right] \quad \text { porque } z_{t} \perp y_{s}(\text { para } s<t) \\
& =0 \quad\left(\text { puesto que } E\left(z_{t}\right)=0\right)
\end{aligned}
$$

2. Varianza

$$
\begin{aligned}
V\left(y_{t}\right) & =E\left(y_{t}^{2}\right) \\
& =E\left(z_{t}^{2}\right) E\left(\alpha_{0}+\alpha_{1} y_{t-1}^{2}\right) \\
& \left.=\alpha_{0}+\alpha_{1} E\left(y_{t-1}^{2}\right), \quad \text { (puesto que } E\left(z_{t}^{2}\right)=1\right)
\end{aligned}
$$

Esto se tiene para todo t. Tomando $E\left(y_{t-1}^{2}\right)$ en función de $E\left(y_{t-2}^{2}\right)$, se tiene:

$$
E\left(y_{t}^{2}\right)=\alpha_{0}+\alpha_{1}\left[\alpha_{0}+\alpha_{1} E\left(y_{t-2}^{2}\right)\right]
$$

De forma analoga, regresando k-veces hacia el pasado y dado que $\alpha_{1}<1$ te-nemos:

$$
E\left(y_{t}^{2}\right)=\sum_{j=0}^{k-1} \alpha_{0} \alpha_{1}^{j}+\alpha_{1}^{k} E\left(y_{t-k}^{2}\right)
$$

Llevando $k \longrightarrow \infty$ la ecuación anterior se convierte en

$$
E\left(y_{t}^{2}\right)=\alpha_{0}\left(\sum_{j=0}^{\infty} \alpha_{1}^{j}\right)
$$


Por lo tanto

$$
E\left(y_{t}^{2}\right)=\alpha_{0} /\left(1-\alpha_{1}\right)
$$

\subsubsection{Función de autocovarianza}

La conclusión final, a partir de los resultados de la sección 3.1.1 y de la presente sección es que un proceso proceso $\mathrm{ARCH}(1)$, cumple con las condiciones de un ruido blanco (media cero y varianza constante).

En adelante $\Psi_{t-1}$ denota la $\sigma$ - algebra generada por $\left\{y_{t-1}, y_{t-2}, y_{t-3}, \ldots\right\}$ es decir $\Psi_{t-1}$ es la información observada hasta el punto $t-1$.

De las ecuaciones (1) y (2) tenemos $y_{t}=z_{t} h_{t}^{1 / 2}$ con $z_{t} \sim N I D(0,1)$ y $h_{t}=$ $\alpha_{0}+\alpha_{1} y_{t-1}^{2}$, entonces:

$$
\begin{aligned}
y_{t}^{2} & =z_{t}^{2}\left(\alpha_{0}+\alpha_{1} y_{t-1}^{2}\right) \\
& \left.=\alpha_{0} z_{t}^{2}+\alpha_{1} z_{t}^{2} y_{t-1}^{2}, \quad \text { (para todo } \mathrm{t}, y_{t-1}^{2}=\alpha_{0} z_{t-1}^{2}+\alpha_{1} z_{t-1}^{2} y_{t-2}^{2}\right)
\end{aligned}
$$

Reemplazando $y_{t-1}^{2}$ en $y_{t}^{2}$, se obtiene:

$$
\begin{aligned}
y_{t}^{2} & =\alpha_{0} z_{t}^{2}+\alpha_{1} z_{t}^{2}\left[\alpha_{0} z_{t-1}^{2}+\alpha_{1} z_{t}^{2} y_{t-2}^{2}\right] \\
& =\alpha_{0} z_{t}^{2}+\alpha_{1} \alpha_{0} z_{t}^{2} z_{t-1}^{2}+\alpha_{1}^{2} z_{t}^{2} z_{t-1}^{2} y_{t-2}^{2} \\
& =\ldots, \text { continuando la recursión en } y_{t-k} \\
& =\alpha_{0} \sum_{j=0}^{k} \alpha_{1}^{j} z_{t}^{2} z_{t-1}^{2} \ldots z_{t-j}^{2}+\alpha_{1}^{k+1} z_{t}^{2} z_{t-1}^{2} \ldots z_{t-k}^{2} y_{t-k-1}^{2}
\end{aligned}
$$

Tomando limite cuando $\mathrm{k} \longrightarrow \infty$, tenemos:

$$
y_{t}^{2}=\alpha_{0} \sum_{j=0}^{\infty} \alpha_{1}^{j} z_{t-1}^{2} \ldots z_{t-j}^{2}
$$

Dadas las ecuaciones (1), (2), (5), tenemos: 


$$
\begin{aligned}
y_{t} & =z_{t}\left(\alpha_{0}+\alpha_{1} y_{t-1}^{2}\right)^{1 / 2} \\
& =z_{t}\left[\alpha_{0}+\alpha_{1} \alpha_{0} \sum_{j=0}^{\infty} \alpha_{1}^{j} z_{t-1}^{2} \ldots z_{t-j}^{2}\right]^{1 / 2} \\
& =z_{t}\left[\alpha_{0}\left(1+\sum_{j=0}^{\infty} \alpha_{1}^{j} z_{t-1}^{2} \ldots z_{t-j}^{2}\right)\right]^{1 / 2} \\
& =z_{t} A_{t-1},
\end{aligned}
$$

donde $A_{t-1}=\left[\alpha_{0}\left(1+\sum_{j=0}^{\infty} \alpha_{1}^{j} z_{t-1}^{2} \ldots z_{t-j}^{2}\right)\right]^{1 / 2}$, se debe notar que $A_{t-1}$ es función de $z_{t-1}, z_{t-2}, \ldots$

De los resultados anteriores la función de autocovarianza entre $y_{t} \mathrm{y} y_{t+h}$, esta dada por:

$$
\text { 3. } \begin{aligned}
\operatorname{Cov}\left(y_{t}, y_{t+h}\right) & =E\left(y_{t} y_{t+h}\right) \\
& =E\left(z_{t+h} z_{t} A_{t-1} A_{t+h-1}\right) \\
& =E\left(E\left(z_{t+h} z_{t} A_{t-1} A_{t+h-1} \mid \Psi_{t+h-1}\right)\right) \\
& =E\left(z_{t} A_{t-1} A_{t+h-1} E\left(z_{t+h} \mid \Psi_{t+h-1}\right)\right) \\
& =0, \quad\left(\text { porque } E\left(z_{t+h} \mid \Psi_{t+h-1}\right)\right)=0
\end{aligned}
$$

De las ecuaciones anteriores tenemos $y_{t} \sim R B\left(0, \sigma_{y}^{2}\right)$, con $\sigma_{y}^{2}=\alpha_{0} /\left(1-\alpha_{1}\right)$, pero $y_{t}$ no es IID, como se verá en la siguiente sección.

\subsubsection{Esperanza y varianza condicional}

A continuación se presenta el desarrollo de la esperanza y varianza condicional del proceso $y_{t}$.

1. Esperanza condicional

$$
\begin{aligned}
E\left(y_{t} \mid \Psi_{t-1}\right) & =E\left(z_{t} \mid \Psi_{t-1}\right)\left(\alpha_{0}+\alpha_{1} y_{t-1}^{2}\right)^{1 / 2} \\
& =E\left(z_{t}\right)\left(\alpha_{0}+\alpha_{1} y_{t-1}^{2}\right)^{1 / 2}, \quad\left(\text { porque } z_{t} \perp y_{s} \quad \text { con } s<t .\right) \\
& =0
\end{aligned}
$$

2. Varianza condicional

$$
\begin{aligned}
V\left(y_{t} \mid \Psi_{t-1}\right) & =E\left(z_{t}^{2} \mid \Psi_{t-1}\right)\left(\alpha_{0}+\alpha_{1} y_{t-1}^{2}\right) \\
& =E\left(z_{t}^{2}\right)\left(\alpha_{0}+\alpha_{1} y_{t-1}^{2}\right) \\
& =\alpha_{0}+\alpha_{1} y_{t-1}^{2}
\end{aligned}
$$

Comunicaciones en Estadística, junio 2009, Vol. 2, No. 1 
De los resultados anteriores se resalta:

- La media es constante en ambos casos, igual a cero.

- La varianza no-condicionada es constante (varianza de largo plazo); mientras que,

- la varianza condicional a corto plazo depende de $y_{t-1}$. Por lo que dados los supuestos, el proceso puede presentar periodos de alta volatilidad o de calma, la varianza a corto plazo no es constante.

\subsubsection{Generalización ARCH(p)}

Los resultados presentados para un $\mathrm{ARCH}(1)$, se pueden generalizar para un proceso $\mathrm{ARCH}(\mathrm{p})$, como se presenta a continuación. El resultado más importante es que un proceso ARCH es un ruido blanco pero no es IID.

En los siguientes items se presentará una generalización de la esperanza y varianza no-condicional, anteriormente presentadas. Teniendo en cuenta las ecuaciones (1) y (2), tenemos:

\section{Esperanza no-condicional}

$$
\begin{aligned}
E\left(y_{t}\right) & =E\left(z_{t} h_{t}^{1 / 2}\right) \\
& =E\left(z_{t}\left(\alpha_{0}+\sum_{i=1}^{p} \alpha_{i} y_{t-i}^{2}\right)^{1 / 2}\right) \\
& =E\left(z_{t}\right) E\left(\alpha_{0}+\sum_{i=1}^{p} \alpha_{i} y_{t-i}^{2}\right)^{1 / 2}, \quad\left(\text { porque } z_{t} \perp y_{s} \quad \text { para todo } s<t\right) \\
& =E\left(z_{t}\right)\left(\alpha_{0}+\sum_{i=1}^{p} \alpha_{i} y_{t-i}^{2}\right)^{1 / 2}, \quad\left(\text { porque } z_{t} \perp y_{s} \quad \text { para todo } s<t\right) \\
& =0
\end{aligned}
$$




\section{Varianza no-condicional}

$$
\begin{array}{rl}
V\left(y_{t}\right) & =E\left(y_{t}^{2}\right) \\
& =E\left(z_{t}^{2}\left(\alpha_{0}+\sum_{i=1}^{p} \alpha_{i} y_{t-i}^{2}\right)\right) \\
& =E\left(z_{t}^{2}\right) E\left(\alpha_{0}+\sum_{i=1}^{p} \alpha_{i} y_{t-i}^{2}\right) \\
& =E\left(\alpha_{0}+\sum_{i=1}^{p} \alpha_{i} y_{t-i}^{2}\right) \\
& =\alpha_{0}+\sum_{i=1}^{p} \alpha_{i} E\left(y_{t-i}^{2}\right) \\
& =\alpha_{0}+\sum_{i=1}^{p} \alpha_{i}\left(\alpha_{0}+\sum_{k=1}^{p} \alpha_{i} E\left(y_{t-i-k}^{2}\right)\right) \\
& =\alpha_{0}+\sum_{i=1}^{p}\left(\alpha_{i} \alpha_{0}+\alpha_{i}^{2} \sum_{k=1}^{p} E\left(y_{t-i-k}^{2}\right)\right) \\
& =\frac{\alpha_{0}}{\sum_{j=1}^{p}\left(\sum_{i=1}^{p} \alpha_{i}\right)^{j}} \\
\alpha_{0} & 1-\sum_{i=1}^{p} \alpha_{i}
\end{array}
$$

Los desarrollos para obtener (a) y (b), se muestran en el añexo 1. En los siguientes items se presentará una generalización de la esperanza y varianza condicional, de nuevo $\Psi_{t-1}$ es la $\sigma$-algebra generada por $\left\{y_{t-1}, y_{t-2}, y_{t-3}, \ldots\right\}$

1. Esperanza condicional

$$
\begin{aligned}
E\left(y_{t} \mid \Psi_{t-1}\right) & =E\left(z_{t} \mid \Psi_{t-1}\right)\left(\alpha_{0}+\sum_{i=1}^{p} \alpha_{i} y_{t-i}^{2}\right)^{1 / 2} \\
& =0
\end{aligned}
$$


2. Varianza condicional

$$
\begin{aligned}
V\left(y_{t}^{2} \mid \Psi_{t-1}\right) & =E\left(z_{t}^{2} \mid \Psi_{t-1}\right)\left(\alpha_{0}+\sum_{i=1}^{p} \alpha_{i} y_{t-i}^{2}\right) \\
& =\alpha_{0}+\sum_{i=1}^{p} \alpha_{i} y_{t-i}^{2} \\
& =h_{t}
\end{aligned}
$$

Las conclusiones para un modelo $\operatorname{ARCH}(p)$ son:

- Un $\mathrm{ARCH}(\mathrm{p})$ es un proceso de ruido blanco pero no es independiente y no es identicamente distribuido.

- Las esperanzas condicional y no-condicional son iguales a cero.

- La varianza no-condicional es constante; mientras que

- la varianza condicional depende de $y_{t-1}, y_{t-2}, \ldots, y_{t-p}$; luego no es fija.

\subsection{Esperanzas y varianzas para un $\operatorname{GARCH}(1,1)$}

\subsubsection{Esperanzas y varianza no-condicional}

Siguiendo con los resultados presentados por Bollerslev (1986), se presenta los desarrollos de la esperanza y varianza en el caso $\operatorname{GARCH}(1,1)$.

1. Esperanza no-condicional

$$
\begin{aligned}
E\left(y_{t}\right) & =E\left(z_{t}\left(\alpha_{0}+\alpha_{1} y_{t-1}^{2}+\beta_{1} h_{t-1}\right)^{1 / 2}\right) \\
& =E\left(z_{t}\right) E\left(\alpha_{0}+\alpha_{1} y_{t-1}^{2}+\beta_{1} h_{t-1}\right)^{1 / 2} \\
& =0
\end{aligned}
$$

2. Varianza no-condicional

$$
\begin{array}{rlr}
V\left(y_{t}\right) & =E\left(y_{t}^{2}\right) \\
& =E\left(z_{t}^{2}\left(\alpha_{0}+\alpha_{1} y_{t-1}^{2}+\beta_{1} h_{t-1}\right)\right) \\
& =E\left(z_{t}^{2}\right) E\left(\alpha_{0}+\alpha_{1} y_{t-1}^{2}+\beta_{1} h_{t-1}\right) \\
& =E\left(\alpha_{0}+\alpha_{1} y_{t-1}^{2}+\beta_{1} h_{t-1}\right) & \\
& \left.=\alpha_{0}+\alpha_{1} E\left(y_{t-1}^{2}\right)+\beta_{1} E\left(h_{t-1}\right)\right) & \text { (c) } \\
& =\frac{\alpha_{0}}{1-\alpha_{1}-\beta_{1}} & \text { (d) }
\end{array}
$$


Los desarrollos para obtener (c) y (d), se presentan en el anexo 2.

\subsubsection{Esperanza y varianza condicional}

1. Esperanza condicional

$$
\begin{aligned}
E\left(y_{t} \mid \Psi_{t-1}\right) & =E\left(z_{t} \mid \Psi_{t-1}\right)\left(\alpha_{0}+\alpha_{1} y_{t-1}^{2}+\beta_{1} h_{t-1}\right)^{1 / 2} \\
& =E\left(z_{t}\right)\left(\alpha_{0}+\alpha_{1} y_{t-1}^{2}+\beta_{1} h_{t-1}\right)^{1 / 2} \\
& =0
\end{aligned}
$$

2. Varianza condicional

$$
\begin{aligned}
V\left(y_{t} \mid \Psi_{t-1}\right) & =E\left(z_{t}^{2} \mid \Psi_{t-1}\right)\left(\alpha_{0}+\alpha_{1} y_{t-1}^{2}+\beta_{1} h_{t-1}\right) \\
& =E\left(z_{t}^{2}\right)\left(\alpha_{0}+\alpha_{1} y_{t-1}^{2}+\beta_{1} h_{t-1}\right) \\
& =h_{t}
\end{aligned}
$$

De los resultados anteriores se puede resaltar que:

1. La esperanza condicional y no-condicionada son iguales a 0 .

2. La varianza de largo plazo es constante, mientras que la varianza de corto plazo puede cambiar.

\subsection{Función de verosimilitud del proceso $\operatorname{ARCH}(1)$}

La estimación de los parametros de un proceso $\mathrm{ARCH}(1) \sqrt{1}$, no se plantea sobre la función de verosimilitud, sino sobre la función de verosimilitud condicional, y dados los suspuestos del modelo $\mathrm{ARCH}(1)$, este tiene distribución condicional normal con media cero y varianza $h_{t}$.

Sea $B_{t}$ la función de densidad de $y_{t}$, dada $\Psi_{t-1}, B_{t}$ esta dada por:

$$
B_{t}=f_{y_{t} \mid \Psi_{t-1}}=\frac{1}{\left(2 \pi h_{t}\right)^{1 / 2}} e^{-\frac{1}{2}\left(h_{t}\right)^{-1} y_{t}^{2}}
$$

La función de verosimilitud condicionada conjunta de T observaciones, está dada por:

$$
B_{1} B_{2} \ldots B_{T}=L=\prod_{t=1}^{T} \frac{1}{\left(2 \pi h_{t}\right)^{1 / 2}} e^{-\frac{1}{2}\left(h_{t}\right)^{-1} y_{t}^{2}}
$$

\footnotetext{
${ }^{1}$ De la misma forma se hace para un proceso $\mathrm{ARCH}(\mathrm{p})$
} 
Maximizar la función anterior es equivalente a maximizar el logaritmo, donde el logaritmo de la función de densidad de la t-esima observación $l_{t}$, esta dado por:

$$
l_{t}=\ln \left(B_{t}\right)=k-\frac{1}{2} \ln h_{t}-\frac{1}{2} \frac{y_{t}^{2}}{h_{t}}, \quad \operatorname{con} k=\ln \frac{1}{(2 \pi)^{1 / 2}}
$$

Y sea $l$ el promedio del logaritmo de la probabilidad, tal que:

$$
\begin{gathered}
l=\frac{1}{T} \sum_{t=1}^{T} l_{t} \\
l_{t}=k-\frac{1}{2} \ln h_{t}-\frac{1}{2} \frac{y_{t}^{2}}{h_{t}}
\end{gathered}
$$

Si notamos $\alpha=\left(\alpha_{0}, \alpha_{1}\right)^{\prime}$, el vector de parámetros, la función $l_{t}$ debe ser maximizada con respecto a $\alpha$. Las condiciones de primer orden y segundo orden(Hessiano), son:

$$
\begin{gathered}
\frac{\partial l_{t}}{\partial \alpha}=\frac{\partial l_{t}}{\partial h_{t}} \frac{\partial h_{t}}{\partial \alpha}=\frac{\partial h_{t}}{\partial \alpha}\left[-\frac{1}{2} \frac{1}{h_{t}}+\frac{y_{t}^{2}}{2 h_{t}^{2}}\right]=\frac{1}{2 h_{t}} \frac{\partial h_{t}}{\partial \alpha}\left[\frac{y_{t}^{2}}{h_{t}}-1\right] \\
M=\frac{\partial^{2} l_{t}}{\partial \alpha \partial \alpha^{\prime}}=-\frac{1}{2 h_{t}} \frac{\partial h_{t}}{\partial \alpha} \frac{\partial h_{t}}{\partial \alpha^{\prime}}\left(\frac{y_{t}^{2}}{h_{t}}\right)+\left[\frac{y_{t}^{2}}{h_{t}}-1\right] \frac{\partial}{\partial \alpha^{\prime}}\left[\frac{1}{2 h_{t}} \frac{\partial h_{t}}{\partial \alpha}\right]
\end{gathered}
$$

Tomando esperanza condicional en (7) tenemos:

$$
\begin{gathered}
E\left(M \mid \Psi_{t-1}\right)=-\frac{1}{2 h_{t}} \frac{\partial h_{t}}{\partial \alpha} \frac{\partial h_{t}}{\partial \alpha^{\prime}} E\left(\frac{y_{t}^{2}}{h_{t}} \mid \Psi_{t-1}\right) \\
+E\left(\left[\frac{y_{t}^{2}}{h_{t}}-1\right] \frac{\partial}{\partial \alpha^{\prime}}\left[\frac{1}{h_{t}} \frac{\partial h_{t}}{\partial \alpha}\right] \mid \Psi_{t-1}\right)
\end{gathered}
$$

Como $E\left(y_{t}^{2} \mid \Psi_{t-1}\right)=h_{t}$, y $h_{t}$ es constante dado $\Psi_{t-1}$ entonces:

$$
E\left(\frac{y_{t}^{2}}{h_{t}} \mid \Psi_{t-1}\right)=1 \Longrightarrow E\left(\left[\frac{y_{t}^{2}}{h_{t}}-1\right] \frac{\partial}{\partial \alpha^{\prime}}\left[\frac{1}{2 h_{t}} \frac{\partial h_{t}}{\partial \alpha}\right] \mid \Psi_{t-1}\right)=0
$$

Así la ecuación (8) es:

$$
E\left(M \mid \Psi_{t-1}\right)=-\frac{1}{2} h_{t} \frac{\partial h_{t}}{\partial \alpha} \frac{\partial h_{t}}{\partial \alpha^{\prime}}
$$

La matriz de información $\Xi$, es simplemente la esperanza negativa del promedio del Hesiano sobre todas las observaciones, entonces : 


$$
\Xi_{\alpha \alpha}=\sum_{t=1}^{T} \frac{1}{2 T} E\left(\frac{1}{h_{t}} \frac{\partial h_{t}}{\partial \alpha} \frac{\partial h_{t}}{\partial \alpha^{\prime}}\right)
$$

su respectivo estimador esta dado por:

$$
\widehat{\Xi}_{\alpha \alpha}=\frac{1}{T} \sum_{t=1}^{T}\left(\frac{1}{2 h_{t}} \frac{\partial h_{t}}{\partial \alpha} \frac{\partial h_{t}}{\partial \alpha^{\prime}}\right)
$$

Ahora si la función $h_{t}$ es de orden lineal en los parámetros, se podría escribir de la forma:

$$
h_{t}=\alpha_{0}+\alpha_{1} y_{t-1}^{2}+\ldots+\alpha_{p} y_{t-p}^{2}
$$

podemos ver que la matriz de información y el vector gradiente tienen una forma simple. Sea $z_{t}=\left(1, y_{t-1}^{2}, \ldots, y_{t-p}^{2}\right)$ y $\alpha^{\prime}=\left(\alpha_{p}, \alpha_{p}, \ldots, \alpha_{p}\right)$, podemos reescribir la función $h_{t}$ descrita como:

$$
h_{t}=z_{t} \alpha, \quad \mathrm{y} \frac{\partial h_{t}}{\partial \alpha}=z_{t}
$$

El vector gradiente será:

$$
\frac{\partial l_{t}}{\partial \alpha}=\frac{1}{2 h_{t}} z_{t}\left[\frac{y_{t}^{2}}{h_{t}}-1\right]
$$

y el estimador de la matriz de información, esta dado por:

$$
\widehat{\Xi}_{\alpha \alpha}=\frac{1}{2 T} \sum_{t=1}^{T}\left(\frac{z_{t}^{\prime} z_{t}}{h_{t}^{2}}\right)
$$

\subsection{Distribución del proceso lineal de primer orden $\mathrm{ARCH}(1)$}

En la búsqueda de determinar las condiciones para que el proceso sea estacionario y encontrar la distribución marginal de los y's, un argumento recursivo es requerido. Los momentos impares son cero por simetría y los momentos pares son calculados usando el siguiente teorema. En todos los casos se asume que el proceso comienza indefinidamente el pasado con $2 \mathrm{r}$ momentos finitos iniciales.

Teorema 1. Para un entero $r$, el $2 r$-ésimo momento para un proceso lineal $A R C H(p)$, con $\alpha_{0}>0, \alpha_{1} \geq 0$ existe si y s'olo si,

$$
\alpha_{1}^{r} \prod_{j=1}^{r}(2 j-1)<1
$$

Comunicaciones en Estadística, junio 2009, Vol. 2, No. 1 
Demostración. Este resultado se mostrará para los casos $r=2, r=3$, y luego se presentará el caso general $r=m$. Sea

$$
w_{t}^{\prime}=\left(y_{t}^{2 r}, y_{t}^{2(r-1)}, \ldots, y_{t}^{2}\right)
$$

Se tiene que $E\left(w_{t} \mid \Psi_{t-1}\right)=\mathbf{b}+\mathbf{D} w_{t-1}$. A continuación, se mostrará que la matriz $\mathbf{D}$ de orden $r \times \mathrm{x}$ es triangular superior y $\mathbf{b}$ es un vector constante de orden $r \times \mathbf{x} 1$. De probabilidad se tiene que dada una variable aleatoria $u \sim N\left(0, \sigma^{2}\right)$ se tiene:

$$
E\left(u^{2 r}\right)=\sigma^{2 r} \prod_{j=1}^{r}(2 j-1)
$$

En particular para el modelo $\mathrm{ARCH}$, si $z_{t} \sim N(0,1)$, entonces:

$$
E\left(z_{t}^{2 r}\right)=\prod_{j=1}^{r}(2 j-1)
$$

Para $r=2$

$$
\begin{gathered}
w_{t}=\left(\begin{array}{c}
y_{t}^{4} \\
y_{t}^{2}
\end{array}\right)=\left(\begin{array}{c}
\left(\alpha_{0}+\alpha_{1} y_{t-1}^{2}\right)^{2} z_{t}^{4} \\
\left(\alpha_{0}+\alpha_{1} y_{t-1}^{2}\right) z_{t}^{2}
\end{array}\right) \\
E\left(w_{t} \mid \Psi_{t-1}\right)=\left(\begin{array}{c}
\left(\alpha_{0}+\alpha_{1} y_{t-1}^{2}\right)^{2} E\left(z_{t}^{4} \mid \Psi_{t-1}\right) \\
\left(\alpha_{0}+\alpha_{1} y_{t-1}^{2}\right) E\left(z_{t}^{2} \mid \Psi_{t-1}\right)
\end{array}\right)
\end{gathered}
$$

aplicando (9a), se tiene que $E\left(z_{t}^{4}\right)=3$. Por lo tanto:

$$
E\left(w_{t} \mid \Psi_{t-1}\right)=\left(\begin{array}{c}
3 \alpha_{0}^{2} \\
\alpha_{0}
\end{array}\right)+\left(\begin{array}{cc}
3 \alpha_{1}^{2} & 6 \alpha_{0} \alpha_{1} \\
0 & \alpha_{1}
\end{array}\right)\left(\begin{array}{c}
y_{t-1}^{4} \\
y_{t-1}^{2}
\end{array}\right)
$$

Para $r=3$

$$
\begin{gathered}
w_{t}=\left(\begin{array}{c}
y_{t}^{6} \\
y_{t}^{4} \\
y_{t}^{2}
\end{array}\right)=\left(\begin{array}{c}
\left(\alpha_{0}+\alpha_{1} y_{t-1}^{2}\right)^{3} z_{t}^{6} \\
\left(\alpha_{0}+\alpha_{1} y_{t-1}^{2}\right)^{2} z_{t}^{4} \\
\left(\alpha_{0}+\alpha_{1} y_{t-1}^{2}\right) z_{t}^{2}
\end{array}\right), \\
E\left(w_{t} \mid \Psi_{t-1}\right)=\left(\begin{array}{c}
\left(\alpha_{0}+\alpha_{1} y_{t-1}^{2}\right)^{3} E\left(z_{t}^{6} \mid \Psi_{t-1}\right) \\
\left(\alpha_{0}+\alpha_{1} y_{t-1}^{2}\right)^{2} E\left(z_{t}^{4} \mid \Psi_{t-1}\right) \\
\left(\alpha_{0}+\alpha_{1} y_{t-1}^{2}\right) E\left(z_{t}^{2} \mid \Psi_{t-1}\right)
\end{array}\right)
\end{gathered}
$$

aplicando (9a)la $E\left(z_{t}^{6}\right)=15$, y desarrollando el polinomio tenemos 


$$
=\left(\begin{array}{c}
15 \\
3 \alpha_{0}^{2} \\
\alpha_{0}
\end{array}\right)+\left(\begin{array}{ccc}
15 \alpha_{1}^{2} & 45 \alpha_{0} \alpha_{1}^{2} & 45 \alpha_{0}^{2} \alpha_{1} \\
0 & 3 \alpha_{1}^{2} & 6 \alpha_{0} \alpha_{1} \\
0 & 0 & \alpha_{1}
\end{array}\right)\left(\begin{array}{c}
y_{t-1}^{6} \\
y_{t-1}^{4} \\
y_{t-1}^{2}
\end{array}\right)
$$

Ahora dado que la distribución condicional de $y$ es normal y aplicando (9a) tenemos:

$$
\begin{aligned}
E\left(y_{t}^{2 m} \mid \Psi_{t-1}\right) & =h_{t}^{2 m} \prod_{j=1}^{r}(2 j-1) \\
& \left.=\left(\alpha_{0}+\alpha_{1} y_{t-1}^{2}\right)^{m} \prod_{j=1}^{r}(2 j-1)\right)
\end{aligned}
$$

Expandiendo esta expresión, se establece que el momento es una combinación lineal de $w_{t-1}$. Luego

$$
\begin{aligned}
E\left(w_{t} \mid \Psi_{t-2}\right) & =E\left[E\left(w_{t} \mid \Psi_{t-1}\right) \mid \Psi_{t-2}\right] \\
& =E\left[\left(b+D w_{t-1}\right) \mid \Psi_{t-2}\right] \\
& =b+D E\left(w_{t-1} \mid \Psi_{t-2}\right) \\
& =b+D\left(b+A w_{t-2}\right) \\
& =b+D b+D^{2} w_{t-2}
\end{aligned}
$$

en general

$$
E\left(w_{t} \mid \Psi_{t-k}\right)=\left(I+D+D^{2}+\ldots+D^{k-1}\right) b+D^{k} w_{t-k}
$$

Dado el supuesto de que la serie comienza indefinidamente en el pasado con $2 r$ momentos, el limite cuando $k$ tiende a infinito existe si y solo si los valores propios de $\mathrm{D}$ se encuentran dentro del circulo unitario.

El limite puede escribirse como:

$$
\lim _{k \rightarrow \infty} E\left(w_{t} \mid \Psi_{t-k}\right)=(I-D)^{-1} b
$$

El cual no depende de las variables condicionales y ni de $t$. Luego, es una expresión para los momentos estacionarios de la distribución condicional de $y$.

$$
E\left(w_{t}\right)=(I-D)^{-1} b
$$

Con lo anterior solamente establecemos que la condición en el teorema es necesario y suficiente tener todos los valores propios dentro del circulo unitario. Como la 
matriz será triangular superior, los elementos de la diagonal son los valores propios. De (7) se tiene que los elementos de la diagonal son simplemente

$$
\alpha_{1}^{m} \prod_{j=1}^{m}(2 j-1)=\prod_{j=1}^{m} \alpha_{1}(2 j-1) \approx \theta_{m} \quad \text { para } \mathrm{m}=1, \ldots, \mathrm{r}
$$

Si $\theta_{r} \geq 1$, los valores propios no se encuentran en el circulo unitario. También se muestra que si $\theta_{r}<1$, entonces $\theta_{m}<1$ para todo $m<r$. Nótese que $\theta_{m}$ es un producto de $m$ factores que son crecientes de forma monótona. Si el $m$-ésimo factor es más grande que uno, entonces $\theta_{m-1}$ necesariamente mas pequeño que $\theta_{m}$.

Si el $m$-ésimo factor es menor que uno, todos los otros factores también serán menores que uno y luego, $\theta_{m-1}$ también es menor que uno y tiene todos los factores menores que uno. Por lo anterior se establece que una condición necesaria y suficiente para todos los elementos de la diagonal es que sean menores que uno, es decir, $\theta_{r}<1$.

El teorema fue facilmente usado para encontrar el segundo y cuarto momento de un proceso de primer orden. La condición para que la varianza sea finita es simplemente que $\alpha_{1}<1$, mientras que para tener un cuarto momento finito se requiere que $3 \alpha_{1}<1$. Con la verificación de estas condiciones, los momentos pueden ser calculados con la formula $E\left(w_{t} \mid \Psi_{t-k}\right)=(I-D)^{-1} b$

Teorema 2. El proceso lineal $A R C H(p)$, con $\alpha_{0}>0, \alpha_{1}, \ldots, \alpha_{p} \geq 0$ tiene covarianza estacionaria si y solo si, la ecuación caracteristica asociada, tiene todas las raices fuera del circulo unitario. La varianza estacionaria esta dada por $E\left(y_{t}^{2}\right)=\alpha_{0} /\left(1-\sum_{j=1}^{p} \alpha_{j}\right)$

Demostración. Sea $w_{t}^{\prime}=\left(y_{t}^{2}, y_{t-1}^{2}, \ldots, y_{t-p}^{2}\right)$

Luego en terminos de la matriz $D$,

$$
E\left(w_{t} \mid \Psi_{t-1}\right)=b+D w_{t-1}
$$

donde $b^{\prime}=\left(\alpha_{0}, 0, \ldots, 0\right) \mathrm{y}$

$$
D=\left[\begin{array}{ccccc}
\alpha_{1} & \alpha_{2} & \ldots & \alpha_{p} & 0 \\
1 & 0 & \ldots & 0 & 0 \\
0 & 1 & \ldots & & 0 \\
0 & 0 & \ldots & 1 & 0
\end{array}\right]
$$

Tomando esperanzas sucesivamente, se obtiene:

$$
E\left(w_{t} \mid \Psi_{t-k}\right)=\left(I+D+D^{2}+\ldots+D^{k-1}\right) b+D^{k} w_{t-k}
$$


Debido a que la serie comienza indefinidamente en el pasado, tiene con varianza finita, si y solo si, todos los valores propios se encuentran dentro del circulo unitario, el limite existe y está dado por:

$$
\lim _{k \rightarrow \infty} E\left(w_{t} \mid \Psi_{t-1}\right)=(I-D)^{-1} b
$$

Como este no depende de condiciones iniciales o de t, este vector es la varianza común para todo t. Esta condición es equivalente a la condicón que todas las raices de la ecuación caracteristica $\left(1-\alpha_{1} z-\ldots-\alpha_{p} z^{p}\right)$, formada por los $\alpha$ 's, se encuentran fuera del circulo unitario. Anderson (1958). Finalmente, el limite de el primer elemento puede ser re-escrito como:

$$
E\left(y_{t}^{2}\right)=\frac{\alpha_{0}}{\left(1-\sum_{j=1}^{p} \alpha_{j}\right)}
$$

\section{Aplicación al caso colombiano}

Ahora se presentará el modelado de la variable Inflación para el caso colombiano, con la metodología que sirvió base de este documento, los datos fueron tomados del Banco de la Republica de Colombia.

La definición más usual de inflación nos dice que inflación es el crecimiento continuo y generalizado de los precios de los bienes y servicios existentes en una economía. Otras definiciones la explican como el movimiento persistente al alza del nivel general de precios o disminución del poder adquisitivo del dinero.

En la figura 1. se aprecian los cambios logarítmicos de la serie de datos correspondiente a la variable inflación donde claramente se aprecia que es estacionaria en media pero no en volatilidad. En ella se observa periodos de calma y periodos de turbulencia. Por lo anterior se aplicará la metodología de Box-Jenkins para ajustar el modelo ARIMA, y posteriormente se complementará con modelos ARCH o GARCH.

En el cuadro 1. se presentan los coeficientes obtenidos para el modelamiento de los retornos logarítmicos de la variable Inflación, el modelo ajustado corresponde a una modelo $A R I M A(24,1,18)$. 
Figura 1: Retornos logarítmicos de la variable Inflación

\begin{tabular}{|l|c|l|c|}
\hline $\mathrm{AR}(1)$ & -0.4773 & $\mathrm{MA}(1)$ & -0.0417 \\
\hline $\mathrm{AR}(4)$ & -0.4706 & $\mathrm{MA}(4)$ & -0.5127 \\
\hline $\mathrm{AR}(12)$ & 0.3592 & $\mathrm{MA}(5)$ & -0.1643 \\
\hline $\mathrm{AR}(13)$ & -0.1512 & $\mathrm{MA}(11)$ & 0.0868 \\
\hline $\mathrm{AR}(17)$ & 0.2532 & $\mathrm{MA}(12)$ & -0.2729 \\
\hline $\mathrm{AR}(19)$ & -0.1064 & $\mathrm{MA}(16)$ & 0.2512 \\
\hline $\mathrm{AR}(22)$ & -0.2222 & $\mathrm{MA}(17)$ & 0.2856 \\
\hline $\mathrm{AR}(24)$ & 0.17377 & $\mathrm{MA}(18)$ & 0.1512 \\
\hline
\end{tabular}

Tabla 1: Coeficientes del modelo ARIMA $(24,1,18)$

En la figura 2. se aprecian los residuales del modelo $A R I M A(24,1,18)$ para la serie Inflación, la cual muestra periodos de baja variabilidad (por ejemplo alrededor de la observación 400) y otros de alta variabilidad (alrededor de la observación 100). Lo anterior es evidencia de no homocedasticidad.

En el cuadro 2. se presentan los coeficientes del modelo ARCH(3). Y finalmente, en la figura 3. se muestra el pronóstico de la variable Inflación, (y sus intervalos de confianza, con el fin de compararlos con el modelo ARCH,) los cuales se obtienen a partir del pronóstico del retorno logarítmico del modelo ARMA estimado. Los intervalos de confianza del pronóstico se obtienen a partir del pronóstico de volatilidad del modelo ARCH (3) estimado. De esta gráfica podemos resaltar el hecho que los intervalos de confianza generados por el modelo ARIMA (li.arma y ls.arma) son superiores a los intervalos generados por el modelo GARCH (li.garch y ls.garch), este comportamiento s'olo se tiene en las primeras observaciones, al correr los pronósticos en tiempo futuro los intervalos se invierten, esto quiere decir, 
Figura 2: Residuales del Modelo $\operatorname{ARIMA}(24,1,18)$ para la variable Inflación

que a medida que pasa el tiempo los intervalos del modelo GARCH son superiores a los intervalos del modelo ARIMA, igual al proceso anterior.

\begin{tabular}{|l|c|c|c|c|c|}
\hline Variable & DF & Estimate & Standard Error & t Value & $\operatorname{Pr}>|t|$ \\
\hline Intercept & 1 & 0.001524 & 0.0000939 & 16.24 & $<.0001$ \\
\hline ARCH0 & 1 & 0.000027 & $9.6172 \mathrm{E}-7$ & 28.20 & $<.0001$ \\
\hline ARCH1 & 1 & 0.5540 & 0.0587 & 9.43 & $<.0001$ \\
\hline ARCH2 & 1 & 0.1927 & 0.0458 & 4.21 & $<.0001$ \\
\hline ARCH3 & 1 & 3.1519 & 0.1280 & 24.63 & $<.0001$ \\
\hline
\end{tabular}

Tabla 2: Coeficientes del modelo ARCH (3)

\section{A. Desarrollo de la varianza no-condicional de un $\operatorname{ARCH}(p)$}

Primero se mostrará el caso $\operatorname{ARCH}(2)$

$$
\begin{aligned}
V\left(y_{t}\right) & =E\left(y_{t}^{2}\right) \\
& =E\left(z_{t}^{2} h_{t}\right) \\
& =E\left(z_{t}^{2}\right) E\left(h_{t}\right) \\
& =E\left(\alpha_{0}+\alpha_{1} y_{t-1}^{2}+\alpha_{2} y_{t-2}^{2}\right)
\end{aligned}
$$

Comunicaciones en Estadística, junio 2009, Vol. 2, No. 1 
Figura 3: Pronóstico de la variable Inflación

De lo anterior tenemos que, para todo $t \rightarrow E\left(y_{t}^{2}\right)=\alpha_{0}+\alpha_{1} E\left(y_{t-1}^{2}\right)+\alpha_{2} E\left(\left(y_{t-2}^{2}\right)\right)$ y en particular para $t-1$ y $t-2$

$E\left(y_{t-1}^{2}\right)=\alpha_{0}+\alpha_{1} E\left(y_{t-2}^{2}\right)+\alpha_{2} E\left(\left(y_{t-3}^{2}\right)\right), \quad \mathrm{y} E\left(y_{t-2}^{2}\right)=\alpha_{0}+\alpha_{1} E\left(y_{t-3}^{2}\right)+\alpha_{2} E\left(\left(y_{t-4}^{2}\right)\right)$

Reemplazando tenemos:

$$
\begin{aligned}
E\left(y_{t}^{2}\right) & =\alpha_{0}+\alpha_{1}\left(\alpha_{0}+\alpha_{1} y_{t-2}^{2} y_{t-3}^{2}\right)+\alpha_{2}\left(\alpha_{0}+\alpha_{1} y_{t-3}^{2}+\alpha_{2} y_{t-4}^{2}\right) \\
& =\alpha_{0}+\alpha_{0}\left(\alpha_{1}+\alpha_{2}\right)+\alpha_{1}^{2} y_{t-2}^{2}+2 \alpha_{1} \alpha_{2} y_{t-3}^{2}+\alpha_{2}^{2} y_{t-4}^{2}
\end{aligned}
$$

Reemplazando $y_{t-2}^{2}, y_{t-3}^{2}, y_{t-4}^{2}$ obtenemos de nuevo

$$
\begin{aligned}
E\left(y_{t}^{2}\right) & =\alpha_{0}+\alpha_{0}\left(\alpha_{1}+\alpha_{2}\right)+\alpha_{0}\left(\alpha_{1}^{2}+\alpha_{1} \alpha_{2}+\alpha_{2}^{2}\right)+\alpha_{1}^{3} y_{t-3}^{2}+3 \alpha_{1}^{2} \alpha_{2} y_{t-4}^{2}+3 \alpha_{1} \alpha_{2}^{2} y_{t-5}^{2}+\alpha_{2}^{3} y_{t-6}^{2} \\
& =\sum_{j=0}^{2} \alpha_{0}\left(\alpha_{1}+\alpha_{2}\right)^{j}+\left(\alpha_{1}^{3}+3 \alpha_{1}^{2} \alpha_{2} B+3 \alpha_{1} \alpha_{2}^{2} B^{2}+\alpha_{2}^{3} B^{3}\right) y_{t-3}^{2} \\
& =\ldots(\text { En general para k }) \\
& =\sum_{j=0}^{k-1} \alpha_{0}\left(\alpha_{1}+\alpha_{2}\right)^{j}+\left(\alpha_{1}+\alpha_{2} B\right)^{k} y_{t-k}^{2}
\end{aligned}
$$

Llevando al limite cuando $k \rightarrow \infty$ 


$$
\begin{aligned}
E\left(y_{t}^{2}\right) & =\sum_{j=0}^{\infty} \alpha_{0}\left(\alpha_{1}+\alpha_{2}\right)^{j} \\
& =\frac{\alpha_{0}}{1-\left(\alpha_{1}+\alpha_{2}\right)}
\end{aligned}
$$

Para el caso $\mathrm{ARCH}(\mathrm{P})$, desarrollará teniendo en cuenta el caso anterior

$$
\begin{aligned}
V\left(y_{t}\right) & =E\left(y_{t}^{2}\right) \\
& =E\left(z_{t}^{2} h_{t}\right) \\
& =E\left(\alpha_{0}+\alpha_{1} y_{t-1}^{2}+\ldots+\alpha_{p} y_{t-p}^{2}\right) \\
& =\alpha_{0}+\alpha_{1} E\left(y_{t-1}^{2}\right)+\ldots+\alpha_{p} E\left(y_{t-p}^{2}\right) \\
& =\alpha_{0}+\alpha_{1}\left(\alpha_{0}+\alpha_{1} E\left(y_{t-2}^{2}\right)+\ldots\right. \\
& +\alpha_{p} E\left(y_{t-p-1}^{2}\right)+\ldots+\alpha_{p}\left(\alpha_{0}+\alpha_{1} E\left(y_{t-p-1}^{2}\right)+\ldots+\alpha_{p} E\left(y_{t-2 p}^{2}\right)\right) \\
& =\alpha_{0}+\alpha_{0} \alpha_{1}+\ldots+\alpha_{0} \alpha_{p}+\alpha_{1}^{2}\left[\alpha_{0}+\alpha_{1} E\left(y_{t-3}^{2}\right)+\ldots+\alpha_{p} E\left(y_{t-p-2}^{2}\right)\right]+\ldots+\alpha_{1} \alpha_{p}\left[\alpha_{0}+\alpha_{1} E\left(y_{t-p-2}^{2}\right)+\ldots+\alpha_{p} E\left(y_{t-p-2}^{2}\right.\right. \\
& =\ldots \text { Iterando } k \text {-veces y tomando el limite cuandok } \rightarrow \infty \\
& =\alpha_{0} \sum_{j=0}^{\infty}\left[\sum_{i=1}^{p} \alpha_{i}\right]^{j} \\
& =\frac{\alpha_{0}}{1-\sum_{i=1}^{p} \alpha_{i}}
\end{aligned}
$$




\section{B. Desarrollo de la varianza no-condicionadal de un $\operatorname{GARCH}(1,1)$}

$$
\begin{aligned}
V\left(y_{t}\right) & =E\left(y_{t}^{2}\right) \\
& =E\left(z_{t}^{2}\left(\alpha_{0}+\alpha_{1} y_{t-1}^{2}+\beta_{1} h_{t-1}\right)\right) \\
& =E\left(z_{t}\right) E\left(\alpha_{0}+\alpha_{1} y_{t-1}^{2}+\beta_{1} h_{t-1}\right) \\
& =E\left(\alpha_{0}+\alpha_{1} y_{t-1}^{2}+\beta_{1} h_{t-1}\right) \\
& \left.=\alpha_{0}+\alpha_{1} E\left(y_{t-1}^{2}\right)+\beta_{1} E\left(h_{t-1}\right)\right) \\
& =\alpha_{0}+\alpha_{1} E\left(\alpha_{0}+\alpha_{1} y_{t-2}^{2}+\beta_{1} h_{t-2}\right)+\beta_{1} E\left(\alpha_{0}+\alpha_{1} y_{t-2}^{2}+\beta_{1} h_{t-2}\right) \\
& =\alpha_{0}+\alpha_{0} \alpha_{1}+\alpha_{0} \beta_{1}+\left[\alpha_{1}^{2}+\alpha_{1} \beta_{1}\right] E\left(y_{t-2}^{2}\right)+\left[\beta_{1}^{2}+\alpha_{1} \beta_{1}\right] E\left(h_{t-2}\right) \\
& =\alpha_{0}+\alpha_{0} \alpha_{1}+\alpha_{0} \beta_{1}+\alpha_{0} \alpha_{1}^{2}+\alpha_{0} \alpha_{1} \beta_{1}+\left[\alpha_{1}^{3}+\alpha_{1}^{2} \beta_{1}\right] E\left(y_{t-3}^{2}\right)+\left[\alpha_{1}^{2} \beta_{1}+\alpha_{1} \beta_{1}^{2}\right] E\left(h_{t-3}^{2}\right)+\alpha_{0} \beta_{1}^{2}+\alpha_{0} \alpha_{1} \beta_{1}+\left[\alpha_{1} \beta_{1}^{2}+\alpha_{1}^{2} \beta\right. \\
& =\ldots \text { Iterando } k-\text { veces y tomando el limite cuandok } \rightarrow \\
& =\alpha_{0}\left[\sum_{j=0}^{\infty}\left(\alpha_{1}+\beta_{1}\right)^{j}\right] \\
& =\frac{\alpha_{0}}{1-\alpha_{1}-\beta_{1}}
\end{aligned}
$$

\section{Agradecimientos}

Agradecimiento muy especial a los pares del articulo, dados los valiosos aportes que a el hicieron.

\section{Referencias}

Anderson, T. W. (1958), An Introduction to Multivariate Statistical Analysis, Jhon Wiley and Sons.

Bollerslev, T. (1986), 'Generalizad autorregresive condicional heterocedasticity.', Journal of Econometrics 31, 307-327.

Box, G. \& Jenkins, G. (1976), Introduction to Time Series, Prentice Hall.

Engle, R. F. (1982), 'Autorregresive conditional heterocedasticity with estimates of the variance of the united kingdom inflation', Econométrica 50, 987-1007. 